\title{
openheart Prognostic impact of postprocedure stroke volume in patients with low- gradient aortic stenosis
}

\author{
Yugo Nara, ${ }^{1}$ Akihisa Kataoka, ${ }^{1}$ Yusuke Watanabe, ${ }^{1}$ Nakashima Makoto, ${ }^{1}$ \\ Hirofumi Hioki, ${ }^{1}$ Hideyuki Kawashima, ${ }^{1}$ Nagura Fukuko, ${ }^{1}$ Ken Kozuma, ${ }^{1}$ \\ Shinichi Shirai, ${ }^{2}$ Norio Tada, ${ }^{3}$ Motoharu Araki, ${ }^{4}$ Toru Naganuma, ${ }^{5}$ \\ Futoshi Yamanaka, ${ }^{6}$ Hiroshi Ueno, ${ }^{7}$ Minoru Tabata, ${ }^{8}$ Kazuki Mizutani, ${ }^{9}$ \\ Akihiro Higashimori, ${ }^{10}$ Kensuke Takagi, ${ }^{11}$ Masanori Yamamoto, ${ }^{12,13}$ \\ Kentaro Hayashida ${ }^{14}$
}

To cite: Nara Y, Kataoka A, Watanabe Y, et al. Prognostic impact of postprocedure stroke volume in patients with lowgradient aortic stenosis. Open Heart 2019;6:e000988.

doi:10.1136/

openhrt-2018-000988

Received 7 December 2018 Revised 26 March 2019 Accepted 6 May 2019
Check for updates

(C) Author(s) (or their employer(s)) 2019. Re-use permitted under CC BY-NC. No commercial re-use. See rights and permissions. Published by BMJ.

For numbered affiliations see end of article.

\section{Correspondence to}

DrAkihisa Kataoka; kataoaki@ sd5.so-net.ne.jp

\section{ABSTRACT}

Objective The effect of postoperative blood flow status on the prognosis of patients with low-gradient severe aortic stenosis (AS) has not been examined. Severe AS is associated with a higher mortality rate after transcatheter aortic valve implantation (TAVI). We examined the prognostic value of low-flow status by comparing stroke volume indices (SVi) before and after TAVI in patients with symptomatic, low-gradient severe AS.

Methods A total of 1613 patients with severe symptomatic AS who underwent TAVI in 14 Japanese institutes for low-gradient severe AS (418 patients, median age 84 years, $32.5 \%$ men) were prospectively enrolled. The primary endpoint was cardiovascular mortality during follow-up after TAVI, and independent predictors were evaluated. Receiver operating characteristic curves were generated to determine the optimal cut-off value of postTAVI SVi for predicting cardiovascular mortality, and the receiver operating characteristic curves of pre-TAVI and post-TAVI SVi were compared.

Results The cardiovascular mortality rate was $4.1 \%$ (17 patients) during follow-up (median 9.2 months). Multivariate analysis revealed post-TAVI SVi to be an independent predictor of cardiovascular mortality (per $10 \mathrm{~mL} / \mathrm{m}^{2}$ decrease; HR, 2.0; $95 \% \mathrm{Cl} 1.28$ to 3.12). The optimal cut-off value of post-TAVI SVi was $41.4 \mathrm{~mL} / \mathrm{m}^{2}$. Post-TAVI SVi showed significantly larger area under the curve than pre-TAVI SVi $(0.74(95 \% \mathrm{Cl} 0.69$ to 0.79$)$ vs 0.61 (95\% $\mathrm{Cl} 0.56$ to 0.65$), \mathrm{p}<0.05)$.

Conclusions Post-TAVI SVi is a better predictor of cardiovascular mortality than pre-TAVI SVi in patients with symptomatic low-gradient severe AS. Low-flow and lownormal-flow status $\left(35 \leq \mathrm{SVi}<40 \mathrm{~mL} / \mathrm{m}^{2}\right)$ require careful management after TAVI.

\section{INTRODUCTION}

Low-gradient severe aortic stenosis (AS), which is classified into classical low-flow, paradoxical low-flow and normal-flow low-gradient AS, is associated with a higher mortality rate even after transcatheter aortic valve implantation (TAVI) than high-gradient severe AS. ${ }^{12}$

\section{Key questions}

What is already known about this subject?

- A low gradient is an independent predictor for mortality after transcatheter aortic valve implantation (TAVI) in severe aortic stenosis (AS).

- Low-flow status has a poorer prognosis compared with the normal-flow status in low-gradient severe AS.

- Studies have demonstrated that TAVI improves left ventricular function, and the improved left ventricular ejection fraction is associated with a good prognosis after TAVI.

- However, in low-gradient severe AS, the type of lowflow status after TAVI that could influence prognosis has not been determined.

What does this study add?

- The stroke volume index (SVi) of post-TAVI was an independent predictor of cardiovascular mortality (HR, 2.00; $95 \% \mathrm{Cl} 1.28$ to 3.12; $\mathrm{p}=0.002$ ) and hospitalisation for heart failure (HR, $1.35 ; 95 \% \mathrm{Cl} 1.03$ to $1.76 ; p=0.028$ ).

- Post-TAVI SVi was a better predictor than pre-TAVI SVi for the estimation of cardiovascular mortality (0.61 (95\% Cl 0.56 to 0.65$)$ vs 0.74 ( $95 \% \mathrm{Cl} 0.69$ to $0.78), p=0.012)$.

How might this impact on clinical practice?

- Clinicians might consider evaluating SVi in patients with low-gradient severe AS before and after TAVI.

- Strict follow-up guidelines should be adopted for patients with persisting low-flow status after TAVI.

Our data also showed that a low-flow status was an independent predictor of cardiovascular mortality after TAVI. ${ }^{3}$ However, it has not yet been examined whether post-TAVI flow status affects the prognosis, especially in patients with low-gradient severe AS. Therefore, we examined the prognostic value of the low-flow status using stroke volume index 


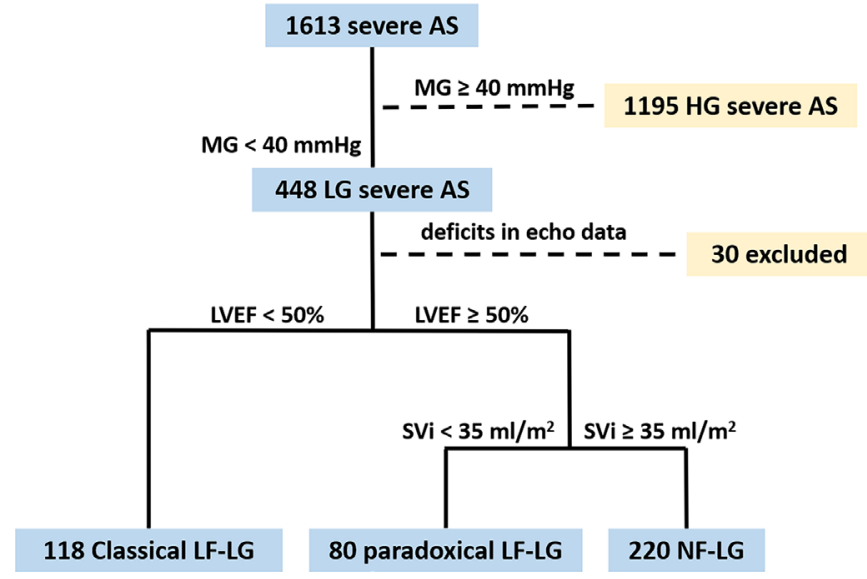

Figure 1 Patient selection process in the present study. The flow chart shows grouping of patients based on the 2014 American Heart Association/American College of Cardiology valvular disease guideline. AS, aortic stenosis; HG, highgradient; LF, low-flow; LG, low-gradient; LVEF, left ventricular ejection fraction; MG, mean gradient; NF, normal-flow; SVi, stroke volume index.

(SVi) before and after TAVI in patients with low-gradient severe AS.

\section{METHODS}

\section{Study population}

From October 2013 to July 2016, a total of 1613 consecutive patients with severe symptomatic AS who underwent TAVI with the Edwards Sapien XT, Edwards Sapien 3 (Edwards Lifesciences, Irvine, California) or Medtronic CoreValve prosthesis (Medtronic, Minneapolis, California) were enrolled prospectively in the Optimized Catheter Valvular Intervention-TAVI registry. The Optimized Catheter Valvular Intervention-TAVI registry is an ongoing Japanese prospective registry that aims to evaluate and observe the background and outcome in patients who undergo TAVI. ${ }^{3-5}$ Fourteen institutions contributed to this registry. The inclusion criteria included the following: (1) presence of symptoms, (2) severe AS with class $\geq$ II (classification by New York Heart Association) and (3) aortic valve area $<1.0 \mathrm{~cm}^{2}$ (or an effective orifice area index $<0.6 \mathrm{~cm}^{2} / \mathrm{m}^{2}$ ). A total of 448 patients with low-gradient severe AS (aortic valve mean gradient $<40$ $\mathrm{mm} \mathrm{Hg}$ ) were included in this study. Of the 448 patients with low-gradient severe AS, 30 patients were excluded because of the deficits in preprocedure or postprocedure echocardiographic data for calculating SVi. Therefore, 418 symptomatic patients were finally included in the present study (figure 1). There was no conversion to surgical aortic valve replacement among these 418 patients. All patients provided written informed consent before participating in this study.

\section{Echocardiographic measurement}

All patients in this study underwent standard two-dimensional B-mode and Doppler transthoracic echocardiography before and after the TAVI procedure. Conventional parameters were measured according to the European Association of Cardiovascular Imaging recommendations. $^{6} 7$ Post-TAVI echocardiography was performed during hospitalisation within a week after TAVI. Patients with low-gradient severe AS (418 patients) were divided into three groups according to pre-TAVI SVi and left ventricular ejection fraction values. ${ }^{2}$ The three groups were formed as follows: classical low-flow low-gradient severe AS (left ventricular ejection fraction $<50 \%$ ), paradoxical low-flow low-gradient severe AS (left ventricular ejection fraction $\geq 50 \%$ and pre-SVi $<35 \mathrm{~mL} / \mathrm{m}^{2}$ ), and normal-flow low-gradient severe AS (left ventricular ejection fraction $\geq 50 \%$ and pre-SVi $\geq 35 \mathrm{~mL} / \mathrm{m}^{2}$ ) (figure 1). Patients' characteristics, echocardiographic parameters, as well as primary and secondary endpoints were compared among the three groups, and pre-TAVI with post-TAVI echocardiographic parameters were compared among each of the three groups.

\section{Study endpoints}

The primary and secondary endpoints included cardiovascular mortality and hospitalisation for heart failure during follow-ups after TAVI. We defined cardiovascular mortality according to the criteria of the Valve Academic Research Consortium-2. ${ }^{8}$ Hospitalisation for heart failure was defined as rehospitalisation after TAVI because of worsening chronic heart failure.

\section{Statistical analysis}

Statistical analyses were performed using SPSS V.24.0 and MedCalc V.17.5.5 software (MedCalc Software, Ostend, Belgium). Continuous variables were assessed for normal distribution using the Shapiro-Wilk test. Data are presented as mean $\pm \mathrm{SD}$ or median and IQR (25th-75th percentiles). We compared the differences in normally distributed data using one-way analysis of variance with subsequent Tukey's honest significant difference test, and the differences in non-normally distributed data using the Kruskal-Wallis test with Mann-Whitney test in the three groups. The Wilcoxon signed-rank test was used to compare pre-TAVI and post-TAVI echocardiographic data. Dichotomous variables are presented as numbers and percentages. $\chi^{2}$ test was used to compare categorical variables. Analyses with Cox proportional hazards regression models were performed to estimate predictors of cardiovascular mortality and hospitalisation for heart failure. Age, sex and clinically relevant echocardiographic variables, with reference to prior studies, were included in the multivariate models using a stepwise variable selection method in which variables were either entered or removed if $\mathrm{p}<0.05$ or $\mathrm{p}>0.1$, respectively. ${ }^{13910}$ The post-TAVI SVi threshold, which optimally predicted primary endpoints, was determined using the Youden Index of the receiver operating characteristic curves. A pairwise comparison of the area under the curve was used to analyse the relationship between cardiovascular mortality, pre-TAVI SVi and post-TAVI SVi. 
Table 1 Patient characteristics and procedure data

\begin{tabular}{|c|c|c|c|c|c|}
\hline & $\begin{array}{l}\text { All patients } \\
(\mathrm{N}=418)\end{array}$ & $\begin{array}{l}\text { Classical LF-LG } \\
(n=118,28.2 \%)\end{array}$ & $\begin{array}{l}\text { Paradoxical LF-LG } \\
(\mathrm{n}=80,19.1 \%)\end{array}$ & $\begin{array}{l}\text { NF-LG } \\
(n=220,52.6 \%)\end{array}$ & $P$ value \\
\hline Age, years & $84.0(81.0-87.0)$ & $84.0(80.0-87.0)$ & $85.0(82.0-88.0)$ & $84.0(81.0-87.0)$ & 0.080 \\
\hline Male & $136(32.5)$ & $48(40.7)$ & $21(26.3)$ & $67(30.5)$ & 0.066 \\
\hline $\mathrm{BSA}, \mathrm{m}^{2}$ & $1.42(1.32-1.53)$ & $1.43(1.34-1.51)$ & $1.41(1.29-1.51)$ & $1.43(1.32-1.56)$ & 0.600 \\
\hline STS score, \% & $7.1(4.9-10.0)$ & $8.6(5.4-13.5) \dagger$ & $7.4(5.3-11.3) \dagger$ & $6.5(4.8-9.1)$ & 0.001 \\
\hline Clinical frailty score & $4.0(3.0-5.0)$ & $4.0(3.0-5.0)$ & $4.0(3.0-4.0)$ & $4.0(3.0-5.0)$ & 0.281 \\
\hline NYHA 3 or 4 & $219(52.4)$ & $70(59.3)$ & $41(51.3)$ & $107(48.6)$ & 0.170 \\
\hline Angina & $70(16.7)$ & $25(21.2)$ & $8(10.0)$ & $37(16.8)$ & 0.118 \\
\hline Syncope & $45(10.8)$ & $10(8.5)$ & $15(18.8)$ & $20(9.1)$ & 0.037 \\
\hline Smoking & 101 (24.2) & $29(24.6)$ & $15(18.8)$ & $57(25.9)$ & 0.437 \\
\hline DM & $143(34.2)$ & $44(37.3)$ & $31(38.8)$ & $68(30.9)$ & 0.318 \\
\hline Dyslipidaemia & $183(43.8)$ & $47(39.8)$ & $32(40.0)$ & $104(47.3)$ & 0.316 \\
\hline Hypertension & $335(80.1)$ & $94(79.7)$ & $62(77.5)$ & $179(81.4)$ & 0.750 \\
\hline CKD & $274(65.6)$ & $79(66.9)$ & $60(75.0)$ & $135(61.4)$ & 0.083 \\
\hline Atrial fibrillation & $136(32.5)$ & $38(32.2)$ & $33(41.3)$ & $65(29.5)$ & 0.160 \\
\hline IHD & $182(43.5)$ & $61(51.7)$ & $33(41.3)$ & $88(40.0)$ & 0.106 \\
\hline PAD & $71(17.0)$ & $22(18.6)$ & $20(25.0)$ & $29(13.2)$ & 0.047 \\
\hline Stroke & $66(15.8)$ & $21(17.8)$ & $9(11.3)$ & $36(16.4)$ & 0.438 \\
\hline COPD & $92(22.0)$ & $25(21.2)$ & $21(26.3)$ & $46(20.9)$ & 0.595 \\
\hline BNP, pg/mL & $215.4(98.1-480.0)$ & $558.3(269.1-953.9)^{*} \dagger$ & $197.7(89.4-346.4)$ & $152.5(71.1-287.4)$ & $<0.001$ \\
\hline Transapical approach & $89(21.3)$ & $20(16.9)$ & $21(26.3)$ & $48(21.8)$ & 0.281 \\
\hline \multicolumn{6}{|l|}{ THV type } \\
\hline Sapien XT & $346(82.8)$ & $94(79.7)$ & 65 (81.3) & $187(85.0)$ & 0.428 \\
\hline CoreValve & $28(6.7)$ & $15(12.7)$ & $4(5.0)$ & $9(4.1)$ & 0.008 \\
\hline Sapien 3 & $44(10.5)$ & $9(7.6)$ & $11(13.8)$ & $24(10.9)$ & 0.373 \\
\hline
\end{tabular}

Values are expressed as $\mathrm{n}(\%)$, median (IQR).

${ }^{*} \mathrm{P}<0.05$ versus paradoxical LF-LG severe AS.

$\dagger P<0.05$ versus NF-LG severe AS.

AS, aortic stenosis; BNP, brain natriuretic peptide; BSA, body surface area; CKD, chronic kidney disease; COPD, chronic obstructive pulmonary disease; DM, diabetes mellitus; IHD, ischaemic heart disease; LF, low-flow; LG, low-gradient;NF, normal-flow; NYHA, New York Heart Association; PAD, peripheral artery disease; STS, Society of Thoracic Surgeons' risk model; THV, transcatheter heart valve.

\section{RESULTS}

\section{Patients' characteristics and echocardiographic parameters}

Patients' characteristics and echocardiographic parameters are shown in tables 1 and 2. The mean age of the patients was 84.0 years (IQR, 81.0-87.0) and $136(32.5 \%)$ were men. The aortic valve mean gradient was $32.4 \mathrm{~mm} \mathrm{Hg}$ (IQR, 28.0-36.0) at baseline, and the transcatheter heart valve mean gradient was $8.5 \mathrm{~mm} \mathrm{Hg}$ (IQR, 7.0-11.0) after TAVI.

\section{Comparison of the three groups}

Classical low-flow low-gradient AS, paradoxical low-flow low-gradient AS and normal-flow low-gradient severe AS groups included 118 (28\%), $80(19 \%)$ and $220(53 \%)$ patients, respectively (figure 1). The normal-flow low-gradient AS group had significantly lower risk score as compared with the other groups as per the Society of Thoracic Surgeons' risk model. Baseline brain natriuretic peptide levels were significantly higher in the classical low-flow low-gradient AS group than that in the paradoxical low-flow low-gradient AS and normal-flow low-gradient severe AS groups. Patients who underwent the CoreValve procedure were most prevalent in the classical low-flow low-gradient AS group. Aortic valve area, mean gradient and maximum velocity of pre-TAVI were highest in the normal-flow low-gradient AS group. Left ventricular ejection fraction values before and after TAVI were lowest in the classical low-flow low-gradient AS group. Pre-TAVI and post-TAVI SVi were lowest in the paradoxical low-flow low-gradient AS group and lower in the classical low-flow low-gradient AS than in the normalflow low-gradient AS group. Pre-TAVI and post-TAVI echocardiographic data showed that the incidence of moderate-severe mitral regurgitation was most prevalent in the classical low-flow low-gradient AS group (table 1).

\section{Midterm clinical outcomes}

All-cause mortality rate during the follow-up period was $12.0 \%$. Among these, the cardiovascular mortality rate 
Table 2 Echocardiography data

\section{All patients}

$(\mathrm{N}=418)$

Classical LF-LG

$(\mathrm{n}=118,28.2 \%)$

\section{Paradoxical LF-LG} $(n=80,19.1 \%)$

NF-LG

$(n=220,52.6 \%)$

$P$ value

TTE (pre-TAVI)

\begin{tabular}{|c|c|c|c|c|c|}
\hline AVA, $\mathrm{cm}^{2}$ & $0.71 \pm 0.15$ & $0.67 \pm 0.15^{\star}$ & $0.63 \pm 0.14^{*}$ & $0.76 \pm 0.13$ & $<0.001$ \\
\hline MG, mm Hg & $32.4(28.0-36.0)$ & $31.6(25.8-35.9)^{\star}$ & $30.5(25.1-34.0)^{\star}$ & $33.8(29.9-37.0)$ & $<0.001$ \\
\hline Vmax, m/s & $3.80(3.50-4.02)$ & $3.68(3.30-4.00)^{\star}$ & $3.70(3.40-3.99)^{\star}$ & $3.88(3.60-4.08)$ & $<0.001$ \\
\hline LVEF, \% & $60.7(47.0-67.0)$ & $39.0(33.2-45.0)^{*} \dagger$ & $64.7(60.3-68.0)$ & $64.5(58.8-70.7)$ & $<0.001$ \\
\hline SVi, mL/m² & $39.7(32.1-49.0)$ & $36.4(28.6-44.9)^{\star} \dagger$ & $27.7(24.8-31.7)^{\star}$ & $45.1(39.7-53.4)$ & $<0.001$ \\
\hline $\mathrm{LAD}, \mathrm{mm}$ & $42.0 \pm 7.3$ & $43.3 \pm 7.7$ & $41.2 \pm 7.0$ & $41.6 \pm 7.1$ & 0.071 \\
\hline$E / e^{\prime}$ & $19.4(14.6-24.3)$ & $21.0(15.5-28.0)^{\star}$ & 17.8 (13.9-25.5) & $18.3(14.3-23.0)$ & 0.035 \\
\hline Moderate-severe AR & $36(8.6)$ & 20 (16.9) & $4(5.0)$ & $12(5.5)$ & 0.001 \\
\hline Moderate-severe MR & $42(10.0)$ & $27(22.9)$ & $6(7.5)$ & $9(4.1)$ & $<0.001$ \\
\hline Mean BP, mm Hg & $106.3(97.5-114.3)$ & $101.7(90.5-108.0)^{\star} \dagger$ & $107.2(100.3-115.4)$ & 108.7 (98.5-117.7) & $<0.001$ \\
\hline LVEDV, mL & 80.9 (62.3-107.5) & $113.0(91.3-142.0)^{*} \dagger$ & $59.0(49.1-72.4)^{\star}$ & $74.6(60.6-99.0)$ & $<0.001$ \\
\hline LVESV, mL & $32.9(20.1-53.9)$ & $68.0(51.0-88.9)^{\star} \dagger$ & $21.0(16.5-25.5)^{*}$ & $26.0(18.9-37.3)$ & $<0.001$ \\
\hline \multicolumn{6}{|l|}{ TTE (post-TAVI) } \\
\hline $\mathrm{EOA}, \mathrm{cm}^{2}$ & $1.60(1.35-1.90)$ & $1.69(1.40-1.98)^{*}$ & $1.50(1.25-1.70) \dagger^{*}$ & $1.63(1.37-1.94)$ & 0.001 \\
\hline THV MG, mm Hg & $8.5(7.0-11.0)$ & $8.00(6.00-10.00) \dagger$ & $8.2(6.3-11.0)$ & $9.0(7.0-11.9)$ & 0.004 \\
\hline LVEF, \% & $61.4(51.4-67.5)$ & $45.3(38.2-53.0)^{*} \dagger$ & $63.9(57.1-68.0)$ & $65.1(59.0-69.6)$ & $<0.001$ \\
\hline SVi, mL/m² & $42.9(34.6-53.0)$ & $41.9(33.4-52.0)^{\star} \dagger$ & $34.3(25.2-40.0) \dagger$ & $46.3(38.6-55.7)$ & $<0.001$ \\
\hline$E / e^{\prime}$ & $19.1(15.0-25.3)$ & $19.5(16.4-27.7)$ & $17.8(13.9-25.4)$ & $19.4(14.8-24.5)$ & 0.264 \\
\hline Moderate-severe PVL & $6(1.4)$ & $3(2.5)$ & $1(1.3)$ & $2(0.9)$ & 0.479 \\
\hline Moderate-severe MR & $33(7.9)$ & $16(13.7)$ & $5(6.3)$ & $12(5.5)$ & 0.024 \\
\hline
\end{tabular}

Values are expressed as $\mathrm{n}(\%)$, median (IQR) and mean $\pm \mathrm{SD}$.

${ }^{*} P<0.05$ versus NF-LG severe AS.

$\dagger P<0.05$ versus paradoxical LF-LG severe AS

$\mathrm{AR}$, aortic regurgitation; AS, aortic stenosis; AVA, aortic valve area;BP, blood pressure; EOA, effective orifice area;E/e', transmitral early peak velocity to early diastolic mitral annular tissue velocity; LAD, left atrial diameter; LF, low-flow; LG, low-gradient; LVEDV, left ventricular end-diastolic volume; LVEF, left ventricular ejection fraction; LVESV, left ventricular end-systolic volume; MG, mean pressure gradient; MR, mitral regurgitation; NF, normal-flow; PVL, paravalvular leakage; SVi, stroke volume index; TAVI, transcatheter aortic valve implantation; THV, transcatheter heart valve; TTE, transthoracic echocardiography; Vmax, max velocity.

was $4.1 \%$. The mean follow-up period after TAVI was 9.2 months (IQR, 3.2-15.3) for the primary endpoint. The rate of hospitalisation for heart failure during follow-up (7.5, IQR, 3.2-14.0 months) was $11.1 \%$ (46 patients). Kaplan-Meier analysis showed no significant difference in the cardiovascular mortality (log-rank test $\mathrm{p}=0.245$ ) and rate of hospitalisation for heart failure (log-rank test $\mathrm{p}=0.129$ ) among the three groups (figure 2 ).

\section{Change between pre-TAVI and post-TAVI echocardiography}

SVi significantly improved after TAVI in the classical low-flow low-gradient AS group and paradoxical low-flow low-gradient AS group (classical low-flow low-gradient AS group, $36.4 \mathrm{~mL} / \mathrm{m}^{2}$ (IQR, 28.6-44.9) vs $41.9 \mathrm{~mL} /$ $\mathrm{m}^{2}$ (IQR, 33.4-52.0), $\mathrm{p}<0.001$; paradoxical low-flow low-gradient AS group, $27.7 \mathrm{~mL} / \mathrm{m}^{2}$ (IQR, 24.8-31.7) vs $34.3 \mathrm{~mL} / \mathrm{m}^{2}$ (IQR, 25.2-40.0), $\mathrm{p}<0.001$ ) (figure $3 \mathrm{~A}$ ). Left ventricular ejection fraction significantly improved after TAVI in the classical low-flow low-gradient AS group (39.0\% (IQR, 33.2-45.0) vs $45.3 \%$ (IQR, 38.2-53.0), $\mathrm{p}<0.001$ ) (figure $3 \mathrm{~B}$ ).

\section{Predictors of clinical outcomes}

The predictors of primary and secondary outcomes are shown in tables 3 and 4 . The independent predictor of

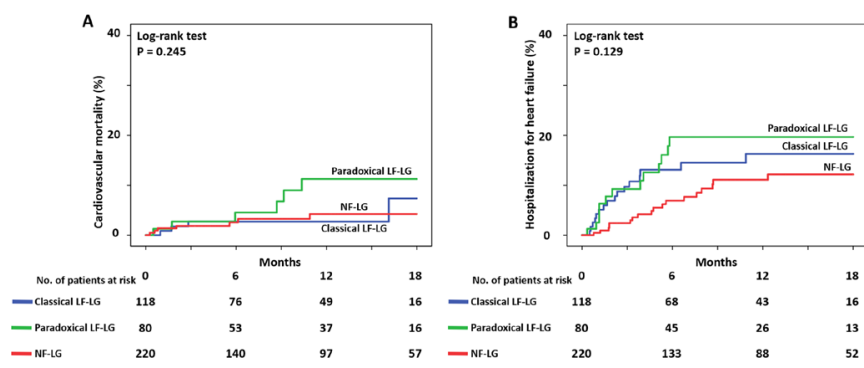

Figure 2 Kaplan-Meier curves for cardiovascular death rate and rate of hospitalisation for heart failure according to the type of LG AS. Survival rates for cardiovascular death $(A)$ and hospitalisation for heart failure $(B)$ were calculated using the Kaplan-Meier method and compared using the log-rank test. No significant differences were observed among the three LG groups. AS, aortic stenosis; LF, low-flow; LG, low-gradient; NF, normal-flow. 
A

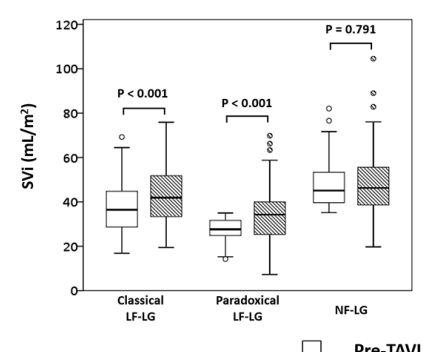

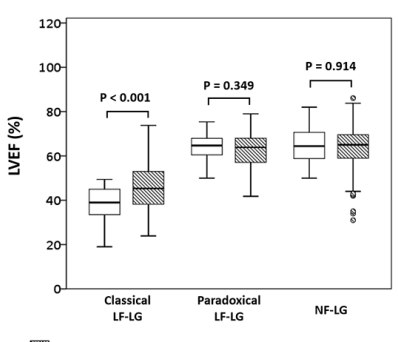

Figure 3 Comparison of echocardiographic parameters before and after TAVI. (A) SVi significantly improved after TAVI in patients with classical and paradoxical LF-LG severe AS, but not in those with HF-LG severe AS. (B) LVEF improved only in patients with classical LF-LG severe AS. AS, aortic stenosis; LF, low-flow; LG, low-gradient; LVEF, left ventricular ejection fraction; NF, normal-flow; SVi, stroke volume index; TAVI, transcatheter aortic valve implantation.

cardiovascular mortality during follow-up was post-TAVI $\mathrm{SVi}$ (per $10 \mathrm{~mL} / \mathrm{m}^{2}$ decrease) (HR, 2.00; 95\% CI 1.28 to 3.12; $\mathrm{p}=0.002)$. The adjusted values for all parameters are shown in table 3 . In addition, the independent predictors of the rate of hospitalisation for heart failure are postTAVI SVi (per $10 \mathrm{~mL} / \mathrm{m}^{2}$ decrease) (HR, 1.35; $95 \%$ CI 1.03 to $1.76 ; \mathrm{p}=0.028)$, pre-TAVI mitral valve regurgitation grade 3 or 4 (HR, 2.71; 95\% CI 1.26 to 5.82 ; $\mathrm{p}=0.011$ ) and baseline natriuretic peptide levels of the brain (per $100 \mathrm{pg} / \mathrm{mL}$ increase) (HR, $1.06 ; 95 \%$ CI 1.01 to 1.11 ; $\mathrm{p}=0.025)$. The adjusted values for all parameters are shown in table 4.

\section{Analysis of receiver operating characteristic curve}

Pairwise comparison of receiver operating characteristic curves showed that post-TAVI SVi presented a significantly larger area under the curve than pre-TAVI SVi for the prediction of cardiovascular mortality (0.61 (95\% CI

Table 3 Predictors of cardiovascular death

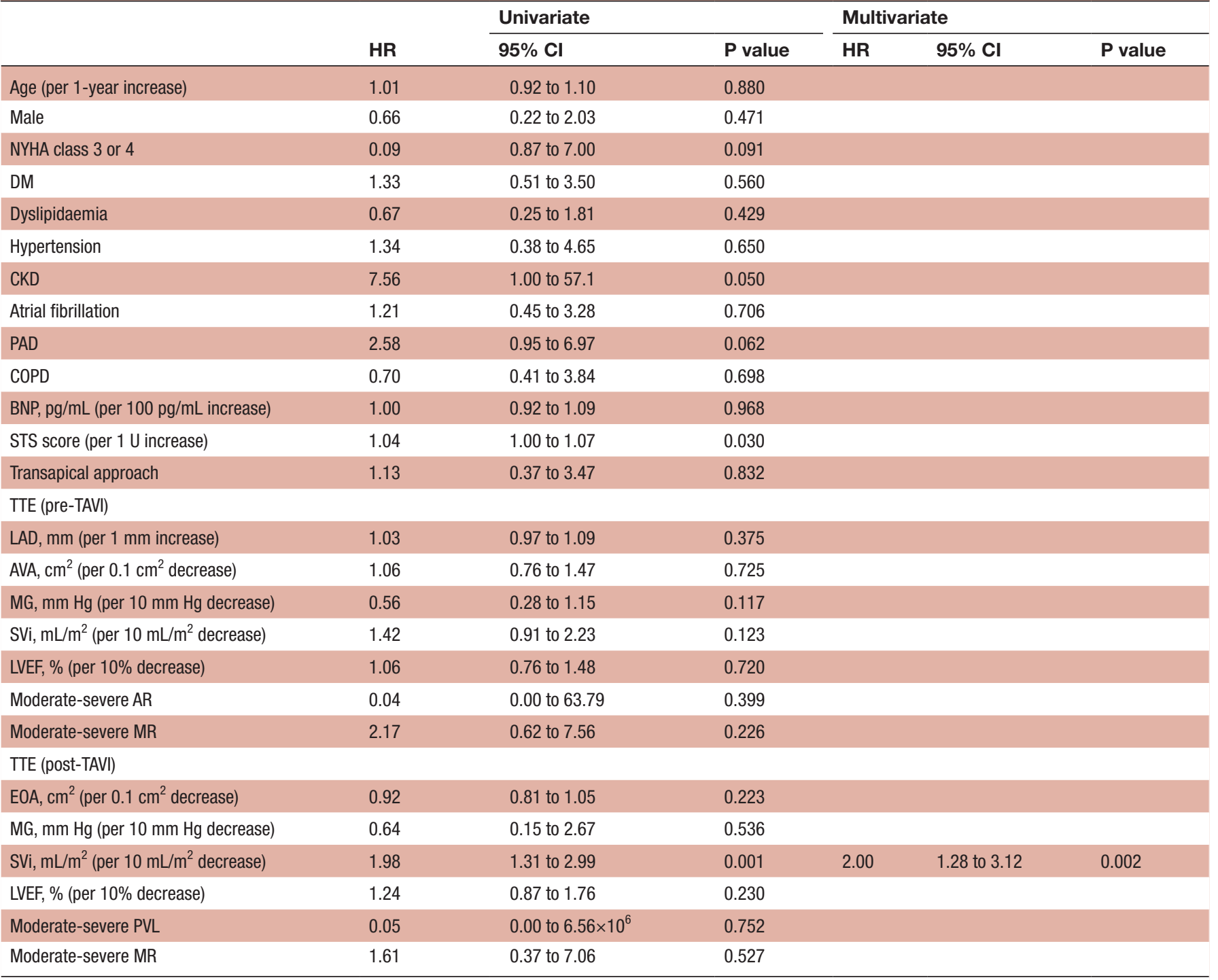

$\mathrm{AR}$, aortic regurgitation; $\mathrm{AVA}$, aortic valve area; BNP, brain natriuretic peptide; $\mathrm{CKD}$, chronic kidney disease; COPD, chronic obstructive pulmonary disease; DM, diabetes mellitus; EOA, effective orifice area; LAD, left atrial diameter; LVEF, left ventricular ejection fraction; MG, mean pressure gradient; MR, mitral regurgitation; NYHA, New York Heart Association; PAD, peripheral artery disease; PVL, paravalvular leakage; STS, Society of Thoracic Surgeons' risk model; SVi, stroke volume index; TAVI, transcatheter aortic valve implantation; TTE, transthoracic echocardiography. 
Table 4 Predictors of hospitalisation for heart failure

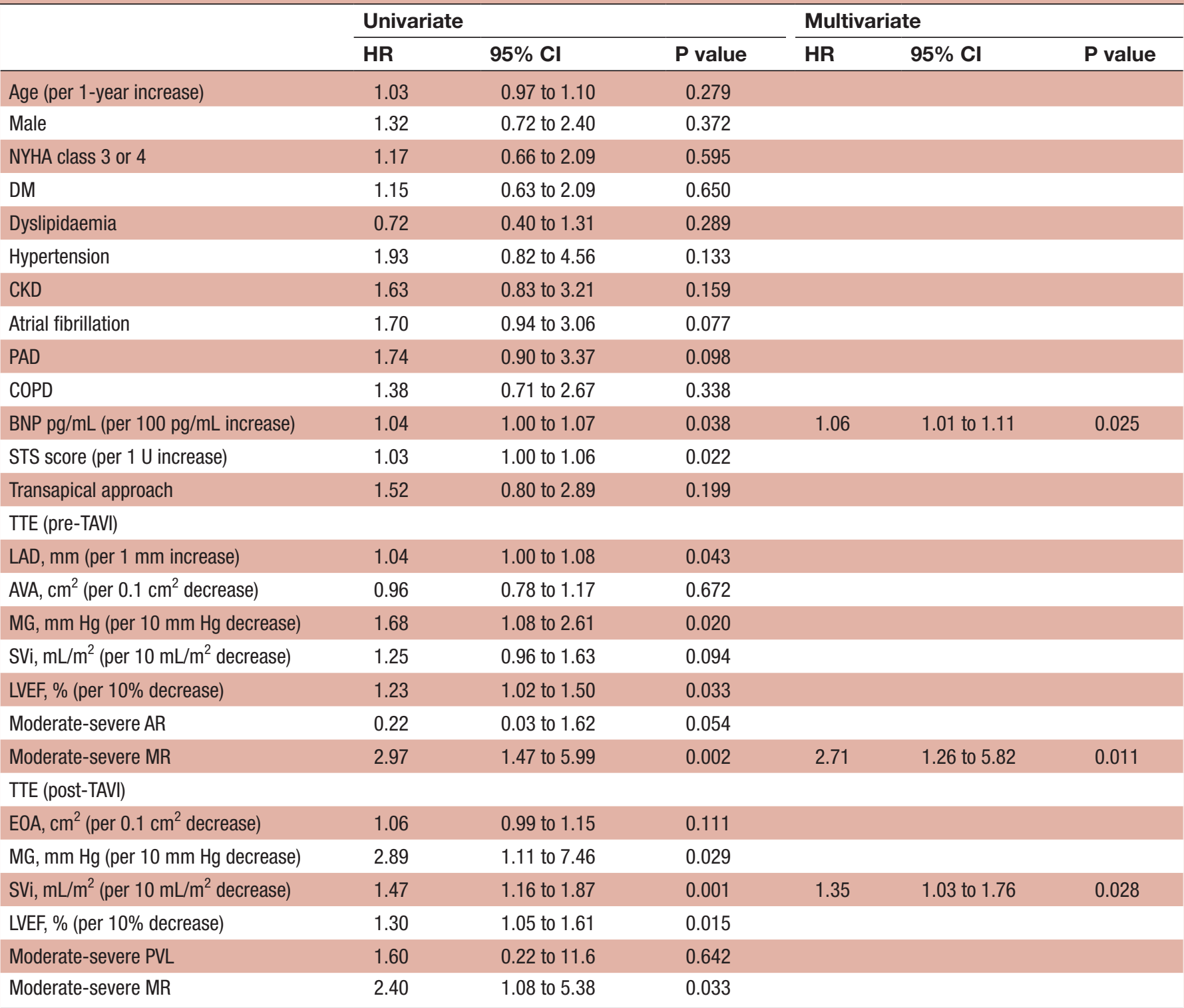

$\mathrm{AR}$, aortic regurgitation; AVA, aortic valve area; BNP, brain natriuretic peptide; CKD, chronic kidney disease; COPD, chronic obstructive pulmonary disease; DM, diabetes mellitus; EOA, effective orifice area; LAD, left atrial diameter; LVEF, left ventricular ejection fraction; MG, mean pressure gradient; MR, mitral regurgitation; NYHA, New York Heart Association; PAD, peripheral artery disease; PVL, paravalvular leakage; STS, Society of Thoracic Surgeons' risk model; SVi, stroke volume index; TAVI, transcatheter aortic valve implantation; TTE, transthoracic echocardiography.

0.56 to 0.65$)$ vs 0.74 (95\% CI 0.69 to 0.78$), \mathrm{p}=0.012)$. A post-TAVI SVi threshold of $<41.4 \mathrm{~mL} / \mathrm{m}^{2}$ was obtained from the receiver operating characteristic curves to predict cardiovascular mortality (sensitivity, $88.2 \%$; specificity, $55.6 \%$ ) (figure 4).

\section{DISCUSSION}

The main findings are as follows: (1) Post-TAVI SVi is an independent predictor of cardiovascular mortality and rate of hospitalisation for heart failure after TAVI in patients with low-gradient severe AS. (2) Post-TAVI $\mathrm{SVi}$ is a better predictor of cardiovascular mortality than pre-TAVI SVi in patients with low-gradient severe AS. (3) Kaplan-Meier analysis showed no significant difference in cardiovascular mortality and rate of hospitalisation for heart failure among the three low-gradient AS groups in this study. (4) Finally, SVi improved after TAVI in the classical and paradoxical low-flow low-gradient severe AS groups, but not in the normal-flow low-gradient severe AS group.

TAVI improved left ventricular function, and the improvement in left ventricular ejection fraction was the predictor of a good prognosis in patients with severe AS who presented with reduced left ventricular ejection fraction. ${ }^{11-14}$ However, Le Ven $e t a l^{15}$ reported that left ventricular ejection fraction underestimates myocardial systolic dysfunction in cases with concentric remodelling. This confirmed our results that left ventricular ejection 


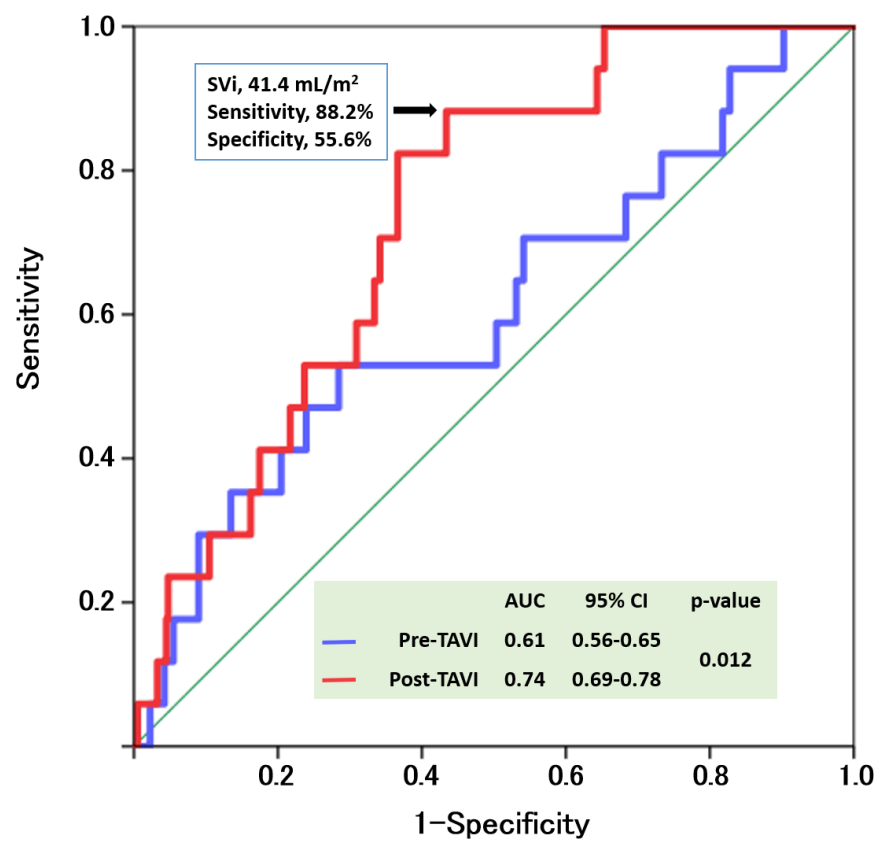

Figure 4 Receiver operating characteristic curves of pre-TAVI and post-TAVI SVi for predicting cardiovascular mortality. Youden Index indicated that the optimal cut-off levels of SVi to predict cardiovascular mortality was 41.4 $\mathrm{mL} / \mathrm{m}^{2}$. Post-TAVI SVi shows significantly larger AUC than pre-TAVI SVi for the prediction of cardiovascular mortality. AUC, area under the curve; SVi, stroke volume index; TAVI, transcatheter aortic valve implantation.

fraction was not a predictor of cardiovascular mortality and rehospitalisation for heart failure.

Prior reports showed that pre-TAVI SVi is a powerful independent predictor of all-cause or cardiovascular mortality, and SVi is a better predictor of a left ventricular ejection fraction prognosis in patients with severe $\mathrm{AS}^{3}{ }^{35-17}$ The present study showed that post-TAVI SVi, not pre-TAVI, is an independent predictor of cardiovascular mortality and rehospitalisation for heart failure. Pairwise comparison of receiver operating characteristic curves also showed that post-TAVI SVi was a better predictor of cardiovascular mortality and rehospitalisation for heart failure than pre-TAVI SVi. The utility of post-TAVI SVi might be because it was influenced by the baseline characteristics and by the effect of TAVI itself.

In our study, Kaplan-Meier analysis showed no significant difference among the three low-gradient groups in cardiovascular mortality and hospitalisation for heart failure. This was not consistent with our prior reports and other reports describing that normal-flow low-gradient severe AS had a better outcome compared with paradoxical low-flow low-gradient severe AS. ${ }^{31819}$ It was considered that there were possibilities that our study population was too small and/or had too short of a follow-up period to produce a significant difference.

In the present study, a post-TAVI SVi threshold of $<41.4$ $\mathrm{mL} / \mathrm{m}^{2}$ was obtained from the receiver operating characteristic curves to predict the cardiovascular mortality, higher than that of pre-TAVI SVi $\left(35 \mathrm{~mL} / \mathrm{m}^{2}\right)$, as defined by the guideline and reported by our previous study. ${ }^{30}$ The patients with low-flow in this study had an increased SVi after TAVI, and naturally the cut-off value was higher than that before TAVI. This suggests that we should carefully consider the patients who show low-flow status $(\mathrm{SVi}$ $\left.<35 \mathrm{~mL} / \mathrm{m}^{2}\right)$ and low-normal-flow status $(35 \leq \mathrm{SVi}<40 \mathrm{~mL}$ / $\left.\mathrm{m}^{2}\right)$ after TAVI.

There are five limitations to our study. First, this was a retrospective analysis of data that were collected prospectively. Second, we did not use core laboratory analysis. The technical inaccuracy and facility disparity of the Doppler echocardiographic measurement of SVi and the mean gradient cannot be ruled out. Third, a small number of events and short-term follow-up, which may limit statistical power, were considered in this study. We selected and analysed the variables using a stepwise (forward selection) method for Cox proportional hazards multivariable regression models to analyse the set of variables in this model. ${ }^{21}$ A larger study with a longer follow-up is strongly needed to confirm these results. Fourth, we did not exclude the five cases of death within 30 days after the procedure, which were procedure-related events. All patients in this study underwent post-TAVI echocardiography, which implied that the intraoperative deaths and deaths immediately after the TAVI procedure were excluded. This could overcome the potential bias of some patients with a clinically probable poor prognosis undergoing echocardiography sooner than other patients. Moreover, post-TAVI echocardiography could exclude at least the earlier events related to the procedure. Finally, we did not measure afterload parameters, such as valvuloarterial impedances both before and after TAVI, which are also considered as prognostic markers after TAVI.

\section{CONCLUSIONS}

SVi measured after TAVI is an independent predictor of cardiovascular mortality in patients with low-gradient severe AS and is a better prognostic marker compared with preprocedure findings. Our results suggest that clinicians should evaluate SVi in patients with low-gradient severe AS before and after TAVI, and patients with low-flow status, especially with low-normal-flow status after TAVI, should be subjected to a stricter follow-up protocol.

\section{Author affiliations}

${ }^{1}$ Division of Cardiology, Department of Internal Medicine, Teikyo University, Tokyo, Japan

${ }^{2}$ Department of Cardiology, Kokura Memorial Hospital, Fukuoka, Japan

${ }^{3}$ Department of Cardiovascular Centre, Sendai Kousei Hospital, Miyagi, Japan

${ }^{4}$ Department of Cardiovascular Medicine, Yokohama City Eastern Hospital, Kanagawa, Japan

${ }^{5}$ Interventional Cardiology Unit, New Tokyo Hospital, Chiba, Japan

${ }^{6}$ Department of Cardiovascular Medicine, Shonan Kamakura General Hospital, Kanagawa, Japan

${ }^{7}$ The Second Department of Internal Medicine, Toyama University Hospital, Toyama, Japan

${ }^{8}$ Department of Cardiology, Tokyo Bay Urayasu Ichikawa Medical Centre, Chiba, Japan

${ }^{9}$ Department of Cardiovascular Medicine, Osaka City University Graduate School of Medicine, Osaka, Japan 
${ }^{10}$ Department of Cardiology, Kishiwada Tokushukai Hospital, Osaka, Japan

${ }^{11}$ Department of Cardiology, Ogaki Municipal Hospital, Gifu, Japan

${ }^{12}$ Division of Cardiovascular Medicine, Toyohashi Heart Center, Aichi, Japan

${ }^{13}$ Division of Cardiovascular Medicine, Nagoya Heart Center, Aichi, Japan

${ }^{14}$ Department of Cardiology, Keio University School of Medicine, Tokyo, Japan

Acknowledgements We are grateful to Miyoshi Sakai for statistical assistance.

Contributors All authors have contributed significantly to the submitted manuscript as follows: study conception and design, data analysis and interpretation, or both: YN, AK and KK; data correction: YW, NM, HH, HK, NF, SS, NT, MA, TN, FY, HU, MT, KM, AH, KT, MY and KH; drafting of the manuscript or critical revision for important intellectual content: YN, AK, YW, SS, NT, MA, TN, FY, HU, MT, $\mathrm{KM}, \mathrm{AH}, \mathrm{KT}$, MY and $\mathrm{KH}$; final approval of the submitted manuscript: all authors.

Funding The Optimized Catheter Valvular Intervention-TAVI registry is supported by Edwards Lifesciences and Medtronic.

Competing interests YW, SS, NT, MA, TN, MY and KH are proctors of transfemoral TAVI for Edwards Lifesciences. YW, SS, TN and MY are proctors of transfemoral TAVI for Medtronic.

\section{Patient consent for publication Obtained.}

Ethics approval This study, which protocol was developed in accordance with the Declaration of Helsinki, was approved by the ethics committee of our institution (number TEIRIN 17-111 and UMIN000020423).

Provenance and peer review Not commissioned; externally peer reviewed. Data availability statement No data are available.

Open access This is an open access article distributed in accordance with the Creative Commons Attribution Non Commercial (CC BY-NC 4.0) license, which permits others to distribute, remix, adapt, build upon this work non-commercially, and license their derivative works on different terms, provided the original work is properly cited, appropriate credit is given, any changes made indicated, and the use is non-commercial. See: http://creativecommons.org/licenses/by-nc/4.0/.

\section{REFERENCES}

1. Baron SJ, Arnold SV, Herrmann HC, et al. Impact of Ejection Fraction and Aortic Valve Gradient on Outcomes of Transcatheter Aortic Valve Replacement. J Am Coll Cardiol 2016;67:2349-58.

2. Clavel M-A, Magne J, Pibarot P. Low-gradient aortic stenosis. Eur Heart J 2016;37:2645-57.

3. Kataoka A, Watanabe Y, Kozuma K, et al. Prognostic impact of low-flow severe aortic stenosis in Small-Body patients undergoing TAVR: the OCEAN-TAVI registry. JACC Cardiovasc Imaging 2018;11:659-69.

4. Nara $\mathrm{Y}$, Watanabe $\mathrm{Y}$, Kozuma K, et al. Incidence, Predictors, and Mid-Term Outcomes of Percutaneous Closure Failure After Transfemoral Aortic Valve Implantation Using an Expandable Sheath (from the Optimized Transcatheter Valvular Intervention [OCEANTAVI] Registry). Am J Cardiol 2017;119:611-7.

5. Hioki H, Watanabe Y, Kozuma K, et al. Pre-procedural dual antiplatelet therapy in patients undergoing transcatheter aortic valve implantation increases risk of bleeding. Heart 2017;103:361-7.
6. Lang RM, Badano LP, Mor-Avi V, et al. Recommendations for cardiac chamber quantification by echocardiography in adults: an update from the American Society of echocardiography and the European association of cardiovascular imaging. Eur Heart $J$ Cardiovasc Imaging 2015;16:233-71.

7. Baumgartner $\mathrm{H}$, Hung J, Bermejo J, et al. Echocardiographic assessment of valve stenosis: EAE/ASE recommendations for clinical practice. Eur J Echocardiogr 2009;10:1-25.

8. Kappetein AP, Head SJ, Généreux P, et al. Updated standardized endpoint definitions for transcatheter aortic valve implantation: the valve academic research Consortium-2 consensus document. Eur Heart J 2012;33:2403-18.

9. Kamperidis V, Joyce E, Debonnaire P, et al. Left ventricular functional recovery and remodeling in low-flow low-gradient severe aortic stenosis after transcatheter aortic valve implantation. J Am Soc Echocardiogr 2014;27:817-25.

10. Clavel M-A, Dumesnil JG, Capoulade R, et al. Outcome of patients with aortic stenosis, small valve area, and low-flow, low-gradient despite preserved left ventricular ejection fraction. J Am Coll Cardiol 2012;60:1259-67.

11. Chen J, Nawaz N, Fox Z, et al. Echocardiographic determinants of LV functional improvement after transcatheter aortic valve replacement. Cathet. Cardiovasc. Intervent. 2016;87:1164-72.

12. Kramer J, Biner S, Ghermezi M, et al. Impact of left ventricular filling parameters on outcome of patients undergoing trans-catheter aortic valve replacement. Eur Heart J Cardiovasc Imaging 2017;18:304-14.

13. Pilgrim T, Wenaweser $P$, Meuli $F$, et al. Clinical outcome of high-risk patients with severe aortic stenosis and reduced left ventricular ejection fraction undergoing medical treatment or TAVI. PLoS One $2011 ; 6$.

14. Schueler R, Sinning J-M, Momcilovic D, et al. Three-dimensional speckle-tracking analysis of left ventricular function after transcatheter aortic valve implantation. J Am Soc Echocardiogr 2012:25:827-34.

15. Le Ven F, Freeman M, Webb J, et al. Impact of low flow on the outcome of high-risk patients undergoing transcatheter aortic valve replacement. J Am Coll Cardiol 2013;62:782-8.

16. Hahn RT, Pibarot P, Stewart WJ, et al. Comparison of transcatheter and surgical aortic valve replacement in severe aortic stenosis: a longitudinal study of echocardiography parameters in cohort a of the partner trial (placement of aortic transcatheter valves). J Am Coll Cardiol 2013;61:2514-21.

17. Hachicha Z, Dumesnil JG, Bogaty P, et al. Paradoxical low-flow, lowgradient severe aortic stenosis despite preserved ejection fraction is associated with higher afterload and reduced survival. Circulation 2007;115:2856-64.

18. Mehrotra $\mathrm{P}$, Jansen $\mathrm{K}$, Flynn AW, et al. Differential left ventricular remodelling and longitudinal function distinguishes low flow from normal-flow preserved ejection fraction low-gradient severe aortic stenosis. Eur Heart J 2013;34:1906-14.

19. Dayan V, Vignolo G, Magne J, et al. Outcome and impact of aortic valve replacement in patients with preserved LVEF and Low-Gradient aortic stenosis. J Am Coll Cardiol 2015;66:2594-603.

20. Nishimura RA, Otto CM, Bonow RO, et al. AHA/ACC guideline for the management of patients with valvular heart disease: a report of the American College of Cardiology/American Heart Association Task Force on practice guidelines. J Am Coll Cardiol 2014;2014:e57-185.

21. Efroymson MA. Multiple regression analysis. In: Ralston A, Wilf HS, eds. Mathematical methods for digital computer. New York, NY: Wiley, 1960: 191-203. 